\title{
Pesquisa
}

\section{RELAÇÃO ENTRE DEPRESSÃO E DEMÊNCIA: UM ENFOQUE NA DOENÇA DE ALZHEIMER}

\author{
Rose Heidy Costa Silva (1) \\ Luane Bitu Leal Alencar (2) \\ Emanuel Tavares Leite Alencar (3) \\ Gislene Farias de Oliveira(4) \\ Rose Anny Costa Silva (5) \\ Analu Bitu Leal Alencar (6)
}

\begin{abstract}
Resumo
A síndrome da demência relaciona-se com a depressão de diversas maneiras e apresenta como características principais o prejuízo da memória, perda de habilidades, além de problemas de comportamento. Sabendo que a doença de Alzheimer (DA) é o tipo mais comum de demência, o presente trabalho objetivou associar depressão e demência, com enfoque na DA e para tal foi realizada uma análise teórica sobre o tema. As causas genéticas, neurobiológicas e sociais estão citadas como principais fatores de risco para o surgimento de depressão concomitante à DA. Cumpre destacar o papel dos familiares no cuidado aos portadores dessa patologia, no entanto, como consequência negativa desse processo, a dinâmica familiar pode sofrer profundas alterações. Foi também percebido que esses pacientes dependem da família no processo de reabilitação e, dessa forma, as abordagens terapêuticas devem atentar para inclusão da família e dos profissionais que assistem esse público.
\end{abstract}

Palavras-chaves: Depressão. Demência. Doença de Alzheimer.

\section{Introdução}

A Demência é uma síndrome, ou seja, é composta por um conjunto de sinais e sintomas que os pacientes manifestam, sendo caracterizada, principalmente, por prejuízo da memória, problemas de comportamento e perda das habilidades. Segundo Balloni (2005), os sintomas mais comuns são o déficit de memória, o qual costuma ser o relato principal ou único na primeira consulta médica, além de problemas com o vocabulário, alterações de humor e de comportamento, dificuldade de executar tarefas domésticas incapacidade de julgar situações, alterações de personalidade e desorientação no tempo e espaço.

A relação entre depressão e demência pode manifestar-se das seguintes maneiras principais: 
depressão na demência, onde o quadro depressivo integra o processo demencial; demência com depressão, consistindo na coexistência de ambos os quadros, sendo que demência precede a depressão; depressão com comprometimento cognitivo, em que neste caso a depressão evolui com alterações cognitivas e demência na depressão, também denominada "pseudodemência depressiva", na qual o comprometimento cognitivo é resultado do processo depressivo (FLORINDO et al, 2002).

A Doença de Alzheimer (DA) é o tipo mais comum de demência (TEIXEIRA-JR e CARAMELLI, 2006). De acordo com Florindo et al. (2002), as pessoas com algum tipo de prejuízo cognitivo representam 5-10\% da população com 65 anos ou mais, e entre esses, mais de 50\% dos casos são devidos à Doença de Alzheimer. Além disso, a prevalência da DA dobra a cada cinco anos em pessoas com idades entre 65 e 85 anos (GREEN, 2001).

Diante do exposto, o presente trabalho pretendeu associar depressão e demência, com enfoque na DA.

\section{Método}

Foi realizada uma revisão sistematizada em busca de artigos utilizando os seguintes descritores: "Doença de Alzheimer", "Depressão" e Demência". A busca foi realizada através dos seguintes banco de dados: SCIELO e GOOGLE ACADÊMICO. A lista de referência dos artigos também foi verificada com o objetivo de localizar artigos considerados relevantes. Dos artigos encontrados, foram selecionados aqueles que se propuseram a relacionar a doença de Alzheimer e demências, em geral, com quadros depressivos.

\section{Fisiopatologia e Etiologia das Doenças Neurodegenerativas em pacientes com depressão}

Os pacientes com Doença de Alzheimer que sofrem depressão frequentemente têm antecedentes familiares de Transtornos Depressivos, o que permite sugerir que os fatores hereditários são importantes na gênese dos sintomas depressivos dos pacientes com Doença de Alzheimer ((FLORINDO et al, 2002).

A presença de alguns fatores pode constituir importantes preditivos para o aparecimento de demência, como existência de síndrome demencial reversível concomitante a episódio depressivo, ocorrência do primeiro episódio depressivo na senescência e presença de anormalidades nos exames de imagem, como atrofia cortical e alargamento de ventrículos (CARTHERY-GOULART, 2007). Foi também encontrado, por meio de exames de imagem, que a hiperintensidade de substância branca frontal se associa com índices mais elevados de depressão em pacientes com demência (BALLONE, 2012). 
O estudo detalhado do encéfalo durante necropsias de pacientes com Doença de Alzheimer e depressão mostraram importantes reduções de bioaminas no locus coeruleus (noradrenalina), núcleos dorsais do rafe (serotonina), e na substância negra (dopamina) (BALLONE, 2012).

Além de causas genéticas e neurobiológicas, existem três categorias de fatores de risco para o surgimento de depressão concomitante à DA, a saber: fatores relacionados à saúde, no qual se encaixa a debilidade cognitiva, principal consequência clínica da DA, além de doenças físicas; fatores sociais, relacionados à exclusão e rejeição tanto por parte dos familiares quanto da sociedade; fatores relacionados à vulnerabilidade pessoal representada por sexo, história de depressão, personalidade prémórbida (NOVARETTI, 2009).

\section{Manifestações clínicas e diagnóstico da síndrome demencial associada à depressão}

Segundo Forlenza (2000), nos estágios avançados da síndrome demencial, o paciente idoso pode ter a capacidade de reconhecer os déficits cognitivos, o que justifica a ocorrência de sintomas depressivo-ansiosos leves ou moderados.

Alguns sinais podem ser identificados, como disforia e apatia, que geralmente estão presentes, além de alterações adicionais como desesperança, sensação de inutilidade e dependência, preocupações somáticas, anedonia e fatigabilidade. Deve-se ficar atento para distúrbio do sono e apetite, pois apesar de cursarem com o processo depressivo, podem estar presentes na síndrome demencial sem depressão (FORLENZA, 2000)

Depressão em pacientes com demência avançada pode ser uma condição difícil de ser diagnosticada, dada à presença de distúrbios de linguagem e comunicação concomitantes (FLORINDO, 2002). Nestes casos, deve-se atentar para a representação comportamental do quadro, avaliando a presença ou não das seguintes manifestações: apatia e retraimento social, lentificação psicomotora, irritabilidade e empobrecimento afetivo, além de agressividade dificuldade de realizar discurso espontâneo. O início do quadro depressivo pode se manifestar através de transformações repentinas do comportamento, ansiedade intensa ou regressão das habilidades funcionais (FORLENZA, 2000).

Dessa forma, entendendo a importância de se diferenciar demência e depressão, sabendo reconhecer quando esses dois quadros ocorrem simultaneamente, faz-se necessário a avaliação do estado mental por meio de testes neuropsicológicos, os quais consistem em avaliar os domínios cognitivos, identificando possíveis déficits, além de mensurar a resposta ao tratamento (MARINHO; LAKS; ENGELHARDT, 2004). Tais testes possuem um roteiro de exame mínimo, tendo os seguintes parâmetros de base: orientação, atenção, memória, aspectos de curto e longo prazo, linguagem, funções visuais e espaciais e capacidade de julgamento e abstração. (CARAMELLI e BARBOSA, 2002) 


\section{Impacto da demência nas relações familiares e entre paciente e profissional de saúde}

A dinâmica familiar pode sofrer profundas alterações devido ao convívio com pacientes demenciados, dado que o cuidado torna-se uma tarefa desgastante, principalmente quando se prolonga por muito tempo. Essa interferência na qualidade de vida dos parentes é materializada pela total dependência da pessoa idosa aos familiares (LEMOS; GAZZOLA; RAMOS, 2006) o que contribui como fator de risco para o a aparecimento do processo depressivo nesses pacientes.

Neri (2002) destaca que apesar das dificuldades enfrentadas pelos familiares, deve-se atentar para o fato de que a assistência aos idosos é uma responsabilidade inerente à família, constituindo, assim, uma norma social.

Dessa forma, a família precisa participar e entender a importância do seu papel como cuidador, embora esse processo acabe gerando impactos em sua vida. Segundo estudo feito por Lemos e col. (2006), os cuidadores de pacientes com DA apresentam altos níveis de impacto subjetivo, sendo esses níveis dependentes de características como nível de escolaridade do cuidador e grau de dependência dos pacientes.

\section{Processo de reabilitação de idosos com demência e depressão}

As doenças neurodegenerativas, próprias da terceira idade, apesar de serem frequentemente negligenciadas pelos profissionais nos estabelecimentos de saúde, possuem diagnóstico diferencial garantido pela avaliação neuropsicológica, método fundamental para detecção precoce, assistência e tratamento dos distúrbios cognitivos (REYS, 2011).

O processo de reabilitação de pacientes com problemas de demência associados à depressão consiste em associar estratégias compensatórias, através de uso de "ajudas externas", como bloco de notas, cartazes, sinalizações (BOTTINO et al, 2002), associadas a práticas de lazer, visto que é constada a relação entre prática de exercícios físicos entre os idosos e menor frequência de depressão (BENEDETTI et al, 2008).

É importante destacar o papel fundamental da família nesse processo, devido à qualidade de vida do idoso depender dos que são responsáveis pelo seu cuidado. Assim, as abordagens terapêuticas devem levar em conta a inclusão da família e dos profissionais que assistem esse público. 


\section{Considerações finais}

Através da análise dos artigos mencionados no presente trabalho, pôde-se perceber que a demência, representada prioritariamente pela Doença de Alzheimer, associada à depressão é bastante prevalente na população idosa. Diante desse conhecimento, faz-se necessário dominar o campo do diagnóstico e do quadro sintomatológico dessas patologias, com intuito de oferecer um cuidado qualificado e um acompanhamento eficaz para esse público, tanto por parte dos familiares quanto dos profissionais de saúde.

\section{Referências}

BALLONE, G.J. Demências. In: PsiqWeb, Internet, disponível em 〈www.psiqweb.med.br $>$, revisto em 2005. Acesso em 10 set. 2012.

BENEDETTI, T.R.B et al. Atividade física e estado de saúde mental de idosos. Rev Saúde Pública. 2008, vol. 42, n. 2, pp. 302-7.

BOTTINO, C.M.C et al. Reabilitação cognitiva em pacientes com Doença de Alzheimer. Arq Neuropsiquiatr. 2002, vol. 60, n.1, pp.70-79.

CARAMELLI, P; BARBOSA, A.T. Como diagnosticar as quatro causas mais freqüentes de demência? Revista Brasileira de Psiquiatria. 2002, vol. 24, n.1, pp. 7-10.

CARTHERY-GOULART, M.T. et al. Versão brasileira da Escala Cornell de depressão em demência (Cornell depression scale in dementia). Arq. Neuro-Psiquiatr. [online]. 2007, vol.65, n.3b, pp. 912-915.

FORLENZA, O.V. Transtornos depressivos na doença de Alzheimer: diagnóstico e tratamento. Rev Bras Psiquiatr. 2000, vol. 22, n.2, pp. 87-95.

GREEN, R.C. Avaliação do Paciente Idoso com Problemas Cognitivos. In: GREEN, R.C. Diagnóstico e tratamento da Doença de Alzheimer e outras demências. $1^{\text {a }}$ ed. Rio de Janeiro: Editora da PUC, 2001, p.21-43.

LEMOS, N.D.; GAZZOLA, J.M.; RAMOS, L.R. Cuidando do paciente com Alzheimer: o impacto da doença no cuidador. Saúde e sociedade. 2006, vol. 15, n. 3, pp. 170-179.

MARINHO, V.M.; LAKS, J.; ENGELHARDT, E. Neuropsiquiatria geriátrica. In: LAKS, J. 
Compêndio de neuropsiquiatria geriátrica. Rio se Janeiro: Guanabara Koogan, 2004. Disponível em: $<$ http://www.portalinclusivo.ce.gov.br/diversos/cartilhasaudeidoso/formao\%20humana\%20em\%20ger iatria\%20e\%20gerontologia\%20-\%20mdulo\%2013.pdf>. Acesso em 15 out. 2012

NERI, A.L. As várias faces do cuidado e do bem estar do cuidador. 1a ed. São Paulo, Editora Alinea, 2002; pp. 9-63.

NOVARETTI, T.M.S. Comparação das habilidades de comunicação na depressão de início tardio e doença de Alzheimer. Tese (doutorado) - Faculdade de Medicina da Universidade de São Paulo. Departamento de Neurologia. São Paulo, 2009.

REYS, B.N. et al. Diagnóstico de demência, depressão e psicose em idosos. Rev Assoc Med Bras. 2006, vol. 52, n. 6, pp. 401-4.

FLORINDO, S. et al. Depressão no Idoso: Diagnóstico, Tratamento e Benefícios da Atividade Física. Vol. 8; n.3, pp. 91-98, Motriz, Rio Claro 2002.

PSIQWEB. Psiquiatria Geral. Disponível em: <http://virtualpsy.locaweb.com.br/ destaque janela.php?cod=101\&cor=\#DCCE8F>. Acesso em 2 nov. 2012.

TEIXEIRA-JR, A.L.; CARAMELli, P. Apatia na doença de Alzheimer. Ver. Bras. Psiquiatr. Vol.28, n.3, pp. 238-241, 2006

\section{Sobre os Autores:}

1. Rose Heidy Costa Silva é Acadêmica de Medicina da Universidade Federal do Ceará - Campus do Cariri.

2. Luane Bitu Leal Alencar é Acadêmica de Medicina da Universidade Federal do Ceará - Campus do Cariri.

3. Emanuel Tavares Leite Alencar é Acadêmico de Medicina da Universidade Federal do Ceará Campus do Cariri.

4. Gislene Farias de Oliveira é Professora na Faculdade de Medicina da Universidade Federal do Ceará campus do Cariri. E-mail: gislenefarias@ gmail.com

5. Rose Anny Costa Silva é Acadêmica de Medicina da Universidade Federal do Ceará - Campus do Cariri.

6. Analu Bitu Leal Alencar é Acadêmica de Medicina da Universidade Federal do Ceará - Campus do Cariri.

\section{Como citar este artigo (Formato ISO):}

SILVA, R.H.C.S.; ALENCAR, L.B.L.; ALENCAR, E.T.L.; OLIVEIRA, G.F.; SILVA, R.A. e ALENCAR, A.B.L.. Relação entre depressão e demência: um enfoque na doença de Alzheimer. Id on Line Revista de Psicologia, Novembro de 2012, vol.1, n.18, p. 96-101. ISSN 1981-1189. 